\title{
Physical health-related quality of life in relation to metabolic health and obesity among men and women in Germany
}

\author{
Julia Truthmann ${ }^{1 *}$ D, Gert B. M. Mensink1', Anja Bosy-Westphal ${ }^{2}$, Ulfert Hapke ${ }^{1}$, Christa Scheidt-Nave ${ }^{1}$
} and Anja Schienkiewitz ${ }^{1}$

\begin{abstract}
Background: This study examined sex-specific differences in physical health-related quality of life (HRQOL) across subgroups of metabolic health and obesity. We specifically asked whether (1) obesity is related to lower HRQoL independent of metabolic health status and potential confounders, and (2) whether associations are similar in men and women.

Methods: We used cross-sectional data from the German Health Interview and Examination Survey 2008-11. Physical HRQoL was measured using the Short Form-36 version 2 physical component summary (PCS) score. Based on harmonized ATPIII criteria for the definition of the metabolic health and a body mass index $\geq 30 \mathrm{~kg} / \mathrm{m}^{2}$ to define obesity, individuals were classified as metabolically healthy non-obese (MHNO), metabolically unhealthy non-obese (MUNO), metabolically healthy obese $(\mathrm{MHO})$, and metabolically unhealthy obese (MUO). Sex-specific analyses including multivariable linear regression analyses were based on PCS as the dependent variable, metabolic health and obesity category as the independent variable with three categories and MHNO as the reference, and age, education, lifestyle and comorbidities as confounders.
\end{abstract}

Results: This study included 6860 participants (3298 men, 3562 women). Compared to MHNO, all other metabolic health and obesity categories had significantly lower PCS in both sexes. As reflected by the beta coefficients [95\% confidence interval] from bivariable linear regression models, a significant inverse association with PCS was strongest for MUO (men: -7.0 [-8.2; -5.8]; women: $-9.0[-10.2 ;-7.9]$ ), intermediate for MUNO (men: $-4.2[-5.3 ;-3$. 1]; women: $-5.6[-6.8 ;-4.4]$ ) and least pronounced for MHO (men: $-2.2[-3.6 ;-0.8]$; women -3.9 [-5.4; -2.5$]$ ). Differences in relation to MHNO remained statistically significant for all groups after adjusting for confounders, but decreased in particular for MUNO (men:-1.3 [-2.3; -0.3]; women: -1.5 [-2.7; -0.3].

Conclusions: Obesity was significantly related to lower physical HRQOL, independent of metabolic health status. Potential confounders including age, educational status, health-related behaviors, and comorbidities explained parts of the inverse relationship. Associations were evident in both sexes and consistently more pronounced among women than men.

Keywords: Metabolically healthy obesity, SF-36, Physical component summary, Sex, Socio-economic status, Lifestyle, Comorbidities, Germany, DEGS1

\footnotetext{
* Correspondence: TruthmannJ@rki.de

${ }^{1}$ Department of Epidemiology and Health Monitoring, Robert Koch Institute,

Berlin, Germany

Full list of author information is available at the end of the article
} International License (http://creativecommons.org/licenses/by/4.0/), which permits unrestricted use, distribution, and reproduction in any medium, provided you give appropriate credit to the original author(s) and the source, provide a link to the Creative Commons license, and indicate if changes were made. The Creative Commons Public Domain Dedication waiver (http://creativecommons.org/publicdomain/zero/1.0/) applies to the data made available in this article, unless otherwise stated. 


\section{Introduction}

Physical health-related quality of life (HRQoL) is a clinically relevant predictor of adverse health outcomes e.g. disability [1], cancer, coronary heart disease and allcause mortality [2]. Obesity has been associated with reduced physical HRQoL in numerous studies $[3,4]$ with a stronger association in women compared to men [5-8]. Several recent studies have demonstrated that even a "metabolically healthy obese" phenotype, i. e. persons with obesity in the absence of metabolic risk factors or comorbidities, show reduced scores of physical HRQoL compared to the group of metabolically healthy non-obese (MHNO) [9-11]. However, these previous studies applied different definitions of metabolic health and HRQoL and considered various subsets of potential confounders. Potential confounders of the association between obesity and lower HRQoL include higher age, low educational status, health-related behaviours [12], and chronic health conditions [13]. In general, metabolic health is associated with higher HRQoL, however, sex-specific associations are still unclear [14]. Previous studies described similar effects among men and women [15] as well as different associations among both sexes, with a negative effect of an impaired health status observed only among women [14]. To our knowledge only one previous study on obesity among metabolic health men and women conducted sex-specific analyses and found that metabolic health had a greater impact on HRQoL than obesity in men while similar effects for metabolic health and obesity were seen in women [9].

Against this background the aim of this study was (1) to determine whether obesity is related to lower HRQoL independent of metabolic health status also considering age, low educational status, health-related behaviours, and comorbidities as potential confounders, and (2) whether associations are similar among men and women.

\section{Methods}

\section{Study design and sample}

The German Health Interview and Examination Survey for Adults 2008-11 (DEGS1) is part of the German health monitoring system. Participants were selected from local population registries in a two-stage sampling procedure. A sample of 7115 participants had participated in the examination part and within this sample representative cross-sectional analyses for the age range of 18-79 years are possible. Exclusion of participants with missing data on body mass index (BMI) and metabolic health status resulted in a study sample of 6860 participants (3298 men, 3562 women). The design of DEGS1 has been previously described in detail [16].

\section{Data collection and study variables}

Physical health-related quality of life

A validated self-administered German language version [17] of the Medical Outcome Short Form-36 version 2 (SF-36 version 2) $[18,19]$ was used to measure physical HRQoL. The questionnaire comprises 36 questions covering eight domains: physical functioning, role-physical, bodily pain, general health, vitality, social functioning, role-emotional and mental health [20]. To improve international comparability "norm-based scoring" was conducted; therefore, the scales were $\mathrm{z}$-transformed using the average values and standard deviations of the 1998 American normative random sample [21]. Two summary measures, the physical component summary (PCS) and the mental component summary combine the information of the eight scales. The present analysis focussed on the PCS score, which correlates mostly with the domains physical functioning, role-physical and bodily pain [20].

\section{Metabolic health and obesity categories}

Body weight, body height and waist circumference (WC) were measured by trained staff [22]. Body weight was measured using a calibrated electronic scale (SECA, column scale 930, Hamburg, Germany) with a precision of $0.1 \mathrm{~kg}$ and body height with a portable stadiometer (Holtain Ltd., UK) with a precision of $0.1 \mathrm{~cm}$. BMI was calculated as body weight divided by height squared and categorized as non-obese (BMI $\left.<30 \mathrm{~kg} / \mathrm{m}^{2}\right)$ and obese $\left(B M I \geq 30 \mathrm{~kg} / \mathrm{m}^{2}\right)$ [23]. WC was measured at the minimal waist using a flexible, non-stretchable tape. Among participants with no visible waist, WC was measured at the midpoint between the lowest rib and the ileac crest. Blood pressure was measured on the right arm following a standardized protocol and using an automated oscillometric device (Datascope Accutorr Plus) [24]. Selfreported physician-diagnosed diseases were obtained in computer-assisted interviews administered by specifically trained physicians [16]. All medications taken in the 7 days prior to the interview were documented by trained medical staff and coded according to the Anatomical Therapeutic Chemical classification system [25]. Blood samples were taken and time of blood draw and number of hours since last meal were recorded [16]. Fasting time was calculated and considered for serum triglyceride cut-off definitions as applied in previous studies [26, 27]. Laboratory analyses were conducted to determine glycated hemoglobin (HbA1c; immunoturbidimetric method, Architect ci8200), triglycerides (enzymatic procedure, Architect ci8200) and high density lipoprotein cholesterol (HDL-C; enzymatic procedure, Architect ci8200).

Metabolic health was defined based on the ATPIII definition [28] as described before for this study population [27] and as fulfilling three or more of the following criteria: 
- WC $<88 \mathrm{~cm}$ in women and $\mathrm{WC}<102 \mathrm{~cm}$ in men

- HbA1c $<5.7 \%$ and had no drug treatment and no physician-diagnosed diabetes

- Blood pressure < 130/85 mmHg and had no drug treatment and no physician-diagnosed hypertension

- Triglycerides $<1.7 \mathrm{mmol} / \mathrm{l}$ (or $<2.1 \mathrm{mmol} / \mathrm{l}$ among participants, who fasted less than $8 \mathrm{~h}$ ) and had no drug treatment and no physiciandiagnosed dyslipidemia

- $\mathrm{HDL}-\mathrm{C} \geq 1.0 \mathrm{mmol} / \mathrm{l}$ in men and $\geq 1.3 \mathrm{mmol} / \mathrm{l}$ in women

Categories of metabolic health status and obesity were defined as metabolically healthy non-obese (MHNO), metabolically unhealthy non-obese (MUNO), metabolically healthy obese (MHO), and metabolically unhealthy obese (MUO).

\section{Covariables}

Covariables were selected to adjust for potential confounders as described in previous studies [9-11]. Educational status (low, middle, high), smoking (no, occasionally, daily), physical activity $\geq 2.5 \mathrm{~h}$ per week (no, yes) and alcohol consumption (never: $0 \mathrm{~g}$, moderate: $0-<10 \mathrm{~g}$ in women and $0-<20 \mathrm{~g}$ in men, high: $\geq 10 \mathrm{~g}$ in women and $\geq 20 \mathrm{~g}$ in men) was assessed by self-administered questionnaires. Comorbidity was defined as reporting at least one of the following physician-diagnosed conditions in personal computer-assisted interviews: cancer, myocardial infarction, stroke asthma, coronary heart disease, chronic heart failure, asthma, musculoskeletal conditions. Based on a question asking for additional diseases requiring current treatment, self-reported gallbladder disease was considered as an obesity-related health condition. Detailed information regarding the definition of covariables is shown in Additional file 1.

\section{Analysis}

In descriptive analyses means, percentages and 95\% confidence intervals were calculated in strata of metabolic health status and obesity categories. All analyses were stratified for sex. Linear regression analyses were performed with the PCS as dependent variable and categories of metabolic health and obesity as independent variable (Model 1). The group of MHNO was used as the reference category. Model 2 was additionally adjusted for age (squared). Model 3 additionally included alcohol consumption, educational status, physical activity, smoking, and comorbidity. In a sensitivity analysis linear regression analyses were performed with the physical functioning score as dependent variable because the association between BMI and HRQoL seems to be most pronounced in this domain [29]. In linear regression analyses gender differences in PCS were identified in categories of metabolic health and obesity. The level of statistical significance was set at $p=0.05$ based on twosided tests. SAS 9.4 survey procedures (SAS Institute Inc., Cary, NC) were used for all statistical analyses. To account for the clustered survey design specific survey procedures were used. Analyses were weighted using a weighting factor to correct for deviations of the net sample from the population structure in Germany and compensate for stratification.

\section{Results}

Table 1 (men) and Table 2 (women) present the study characteristics by strata of metabolic health status and obesity. In men the distribution of participants was MHNO ( $n=1900)$, MUNO $(n=608)$, MHO $(n=196)$, MUO $(n=594)$ and in women it was MHNO $(n=2193)$, MUNO $(n=514)$, MHO $(n=277)$, MUO $(n=578)$. MHNO (mean age men: 42.1 years, women: 42.4 years) were younger than MHO and MUO; MUNO (men: 55.6 years, 59.3 years) was the oldest group. Percentage of low educational status was lowest in MHNO (men: 30.7\%, women: 27.8\%) and highest in MUO (men: $51.3 \%$, women: $59.5 \%$ ). Percentages of daily smoking and physical activity $\geq 2.5 \mathrm{~h}$ were lower among metabolically unhealthy men and women. Percentages of high alcohol consumption were highest among MUNO for men (22.7\%) and highest among MHNO for women (14.9\%). The prevalence of comorbidities was higher among metabolically unhealthy men and women. The PCS was highest among MHNO (men: 54.0, women: 53.1) and lowest among MUO (men: 47.0, women: 44.1).

Table 3 shows the PCS in relation to categories of metabolic health and obesity for men and women. Additionally, Table S1 (Additional file 2) presents the estimations for physical functioning. Compared with MHNO, all other categories of metabolic health and obesity were consistently associated with lower PCS values among men and among women. The inverse association with PCS was strongest for MUO (men: -7.0, women: -9.0), intermediate for MUNO (men: -4.2 , women: -5.6$)$ and least pronounced for MHO (men: -2.2 , women -3.9 . Adjusting for age (Model 2) and further adjustment for sociodemographic characteristics, lifestyle variables and comorbidities (Model 3) decreased the association among all strata of metabolic health and obesity. After adjusting for confounding variables, the effect was strongest for MUO (men: -3.9, women: -4.9), intermediate for MHO (men: -1.8, women: -2.1) and least pronounced for MUNO (men: -1.3 , women: -1.5$)$. The results for the PCS were similar to the results found in sensitivity analyses using the physical functioning score as dependent variable (Additional file 2: Table S1).

Table 4 presents gender differences in PCS for categories of metabolic health and obesity status. PCS 
Table 1 Study characteristics among men (mean/\% and 95\% confidence interval)

\begin{tabular}{|c|c|c|c|c|c|}
\hline & $\mathrm{N}$ missing & $\begin{array}{l}\text { MHNO } \\
N=1900\end{array}$ & $\begin{array}{l}\text { MUNO } \\
N=608\end{array}$ & $\begin{array}{l}\mathrm{MHO} \\
N=196\end{array}$ & $\begin{array}{l}M \cup O \\
N=594\end{array}$ \\
\hline Age (mean, years) & 0 & $42.1(41.3-42.9)$ & $56.8(55.2-58.4)$ & $44.4(42.3-46.6)$ & $55.6(54.2-57.1)$ \\
\hline Educational status (\%) & 21 & & & & \\
\hline Low & & $30.7(27.5-34.0)$ & $41.3(35.8-47.0)$ & $41.2(32.3-50.7)$ & $51.3(45.5-57.2)$ \\
\hline Middle & & $51.3(48.4-54.2)$ & $38.8(33.9-43.8)$ & $48.3(39.3-57.4)$ & $38.3(32.7-44.1)$ \\
\hline High & & $18.0(15.9-20.4)$ & $20.0(16.0-24.6)$ & $10.5(6.7-16.0)$ & $10.4(8.0-13.5)$ \\
\hline Smoking (\%) & 17 & & & & \\
\hline No & & $64.1(60.9-67.2)$ & $74.0(68.1-79.2)$ & $66.8(57.0-75.4)$ & $72.1(66.7-76.9)$ \\
\hline Occasionally & & $7.5(6.2-9.1)$ & $4.3(2.5-7.3)$ & $5.9(3.4-10.3)$ & $5.6(3.6-8.7)$ \\
\hline Daily & & $28.4(25.6-31.4)$ & $21.7(17.0-27.2)$ & $27.3(18.9-37.6)$ & $22.3(17.9-27.6)$ \\
\hline Physical activity $\geq 2.5$ h (\%) & 116 & $28.2(25.5-31.0)$ & $18.4(14.6-22.9)$ & $30.0(22.5-38.6)$ & $18.9(15.0-23.4)$ \\
\hline Alcohol consumption (\%) & 55 & & & & \\
\hline Never & & $10.4(8.4-12.8)$ & $8.4(5.8-11.9)$ & $11.3(6.7-18.3)$ & $9.2(6.2-13.3)$ \\
\hline Moderate & & $72.0(69.1-74.8)$ & $68.9(64.9-72.7)$ & $72.6(63.7-80.0)$ & $71.5(65.9-76.4)$ \\
\hline High & & $17.6(15.6-19.8)$ & $22.7(19.3-26.5)$ & $16.1(10.7-23.4)$ & $19.4(15.4-24.1)$ \\
\hline One or more comorbidities (\%) & 29 & $25.7(23.2-28.3)$ & $51.9(46.8-57.0)$ & $25.7(23.2-28.3)$ & $49.5(44.1-54.9)$ \\
\hline Physical component summary (mean) ${ }^{a}$ & 158 & $54.0(53.6-54.5)$ & $49.8(48.8-50.9)$ & $51.8(50.4-53.3)$ & $47.0(45.9-48.2)$ \\
\hline
\end{tabular}

MHNO metabolically healthy non-obese, $M U N O$ metabolically unhealthy non-obese, $M H O$ metabolically healthy obese, $M U O$ metabolically unhealthy obese

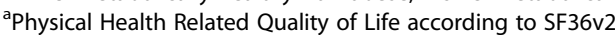

scores were consistently lower among women compared to men in all strata. Gender differences were lowest among MHNO (-0.9) and highest among MUO (-2.9). After adjusting for confounding variables differences remained statistically significant only among MHNO.

\section{Discussion}

Obesity and metabolic aberration alone and in combination were associated with impaired physical HRQoL in both sexes. The effect of both factors in combination corresponds approximately to the effects for obesity and metabolic aberration alone. These results underline

Table 2 Study characteristics among women (mean/\% and 95\% confidence interval)

\begin{tabular}{|c|c|c|c|c|c|}
\hline & $\mathrm{N}$ missing & $\begin{array}{l}\text { MHNO } \\
N=2193\end{array}$ & $\begin{array}{l}\text { MUNO } \\
N=514\end{array}$ & $\begin{array}{l}\mathrm{MHO} \\
N=277\end{array}$ & $\begin{array}{l}M U O \\
N=578\end{array}$ \\
\hline Age (mean, years) & 0 & $42.4(41.7-43.1)$ & $61.9(60.6-63.3)$ & $47.0(45.0-48.9)$ & $59.3(57.8-60.8)$ \\
\hline Educational status (\%) & 17 & & & & \\
\hline Low & & $27.8(25.3-30.6)$ & $55.9(49.9-61.8)$ & $45.1(37.0-53.4)$ & $59.5(53.8-65.0)$ \\
\hline Middle & & $56.2(53.3-59.1)$ & $36.0(30.3-42.1)$ & $49.9(42.0-57.9)$ & $36.5(31.4-41.9)$ \\
\hline High & & $16.0(13.6-18.6)$ & $8.1(6.1-10.7)$ & $5.0(3.1-8.0)$ & $4.0(2.5-6.2)$ \\
\hline Smoking (\%) & 17 & & & & \\
\hline No & & $69.9(67.0-72.7)$ & $78.9(73.4-83.5)$ & $74.5(67.8-80.3)$ & $79.1(74.3-83.2)$ \\
\hline Occasionally & & $7.7(6.4-9.2)$ & $2.1(1.1-4.1)$ & $4.1(2.1-7.7)$ & $1.5(0.7-3.3)$ \\
\hline Daily & & $22.4(20.0-25.0)$ & $19.0(14.8-24.1)$ & $21.4(16.1-27.9)$ & $19.4(15.5-24.1)$ \\
\hline Physical activity $\geq 2.5$ h (\%) & 110 & $15.9(14.1-18.0)$ & $13.7(10.3-18.1)$ & $16.9(12.0-23.2)$ & $14.0(10.5-18.4)$ \\
\hline Alcohol consumption (\%) & 44 & & & & \\
\hline Never & & $16.3(14.5-18.3)$ & $17.0(13.0-21.8)$ & $21.0(15.6-27.8)$ & $29.7(25.1-34.7)$ \\
\hline Moderate & & $68.8(66.2-71.3)$ & $70.8(65.3-75.8)$ & $68.9(60.7-76.1)$ & $61.1(55.8-66.1)$ \\
\hline High & & $14.9(13.1-16.9)$ & $12.2(8.8-16.8)$ & $10.0(5.5-17.4)$ & $9.2(6.2-13.6)$ \\
\hline One or more comorbidities (\%) & 22 & $26.0(23.7-28.6)$ & $54.6(49.4-59.7)$ & $40.2(34.0-46.7)$ & $59.9(54.7-64.9)$ \\
\hline Physical component summary (mean) ${ }^{a}$ & 150 & $53.1(52.7-53.6)$ & $47.6(46.4-48.7)$ & $49.2(47.8-50.6)$ & $44.1(43.1-45.1)$ \\
\hline
\end{tabular}


Table 3 Linear regression models for physical component summary (SF-36 v2) according to metabolic health and obesity status

\begin{tabular}{|c|c|c|c|c|c|c|c|c|c|c|c|c|c|c|}
\hline & \multirow[t]{2}{*}{$N$} & \multirow[t]{2}{*}{ MHNO } & \multicolumn{4}{|c|}{ MUNO } & \multicolumn{4}{|l|}{$\mathrm{MHO}$} & \multicolumn{4}{|l|}{$\mathrm{MUO}$} \\
\hline & & & Beta & Lower CL & Upper CL & $p$ & Beta & Lower CL & Upper CL & $p$ & Beta & Lower CL & Upper CL & $p$ \\
\hline \multicolumn{15}{|l|}{ Men } \\
\hline Model 1 & 3140 & Reference & -4.2 & -5.3 & -3.1 & $<.001$ & -2.2 & -3.6 & -0.8 & .003 & -7.0 & -8.2 & -5.8 & $<.001$ \\
\hline Model 2 & 3140 & Reference & -1.7 & -2.7 & -0.6 & .002 & -1.9 & -3.3 & -0.5 & .006 & -4.7 & -5.9 & -3.5 & $<.001$ \\
\hline Model 3 & 2988 & Reference & -1.3 & -2.3 & -0.3 & .014 & -1.8 & -3.1 & -0.5 & .009 & -3.9 & -5.1 & -2.7 & $<.001$ \\
\hline \multicolumn{15}{|l|}{ Women } \\
\hline Model 1 & 3412 & Reference & -5.6 & -6.8 & -4.4 & $<.001$ & -3.9 & -5.4 & -2.5 & $<.001$ & -9.0 & -10.2 & -7.9 & $<.001$ \\
\hline Model 2 & 3412 & Reference & -2.1 & -3.3 & -0.9 & $<.001$ & -3.3 & -4.7 & -1.9 & $<.001$ & -6.2 & -7.4 & -5.0 & $<.001$ \\
\hline Model 3 & 3270 & Reference & -1.5 & -2.7 & -0.3 & .018 & -2.1 & -3.2 & -1.0 & $<.001$ & -4.9 & -6.1 & -3.6 & $<.001$ \\
\hline
\end{tabular}

Model 1: unadjusted

Model 2: adjusted for age (squared)

Model 3: additionally adjusted for educational status, smoking, physical activity, alcohol consumption, comorbidities

$C L$ confidence limit, $M H N O$ metabolically healthy non-obese, $M U N O$ metabolically unhealthy non-obese, $M H O$ metabolically healthy obese, $M U O$ metabolically unhealthy obese

previous findings suggesting that $\mathrm{MHO}$ individuals are not always completely healthy with regard to HRQoL $[10,11]$ as well as morbidity of cardiovascular diseases $[30,31]$ and all-cause mortality [32, 33]. A recent clinical study found similar levels of HRQoL among MHO and MUO individuals [34]. This finding seems to contrast with our results with larger effects of MUO vs. MHNO compared to $\mathrm{MHO}$ vs. MHNO. The discrepancies may be caused by the clinical setting as patients who are willing to be treated probably are likely to suffer from obesity. However, in conclusion both studies support the finding that $\mathrm{MHO}$ is not a healthy state.

The association between physical HRQoL, metabolic health and obesity categories is stronger among women, as lower PCS values were found in all strata of metabolic health and obesity among women compared to men. Furthermore, differences between strata were greater among women than among men. Lower values of physical HRQoL among women compared to men were also reported in previous studies analysing the association with obesity $[7,8]$ and obesity in combination with metabolic health $[9,10]$ opposed to one meta-analysis where no gender differences were found [3]. In the present study, adjustment for potential confounders including education, health-related behaviours and comorbidities gender differences were attenuated. Thus, lower
HRQoL among women than men might be partially explained by sex-differences in the prevalence of obesityrelated comorbidities [35] and health-related behaviours e.g. physical activity [36]. Among both, men and women physical HRQoL was highest for the MHNO group followed by MHO and MUNO and lowest for the group of MUO. This is in contrast to findings of a Korean cross-sectional study [9], where HRQoL were measured using the euroqol-5 dimensions questionnaire. Among Korean men physical HRQoL was lowest among the group of MUNO, suggesting that an unhealthy metabolic status have a greater impact than obesity. In this previous study, no differences in physical HRQoL were observed between groups of metabolic status and obesity among men after adjusting for age, sociodemographic variables, lifestyle and comorbidity. Among Korean women, the results were similar to the findings of our study.

Persons in the different categories of obesity and metabolic health status substantially differed according to mean age, educational status and prevalence of one or more comorbidities. Previous studies reported lower values of HRQoL at higher age, among persons with chronic diseases, and in low educational status groups $[13,21,37,38]$. Considering these potential confounders in multivariable regression analyses in the present study

Table 4 Gender differences (reference = men) in physical component summary (SF-36 v2) in categories of metabolic health and obesity status

\begin{tabular}{|c|c|c|c|c|c|c|c|c|c|c|c|c|c|c|c|c|}
\hline & \multicolumn{4}{|c|}{ MHNO N = 3945} & \multicolumn{4}{|c|}{ MUNO N = 1064} & \multicolumn{4}{|c|}{$\mathrm{MHO} N=457$} & \multicolumn{4}{|c|}{ MUO N = 1084} \\
\hline & Beta & Lower CL & Upper CL & $p$ & Beta & Lower CL & Upper CL & $p$ & Beta & Lower CL & Upper CL & $P$ & Beta & Lower CL & Upper CL & $p$ \\
\hline Model 1 & -0.9 & -1.5 & -0.2 & .007 & -2.3 & -3.7 & -0.8 & .003 & -2.7 & -4.8 & -0.5 & .014 & -2.9 & -4.5 & -1.4 & $<.001$ \\
\hline Model 2 & -0.8 & -1.5 & -0.2 & .008 & -1.1 & -2.4 & 0.2 & .084 & -2.1 & -4.2 & -0.1 & .043 & -2.3 & -3.7 & -0.8 & .003 \\
\hline Model 3 & -0.8 & -1.4 & -0.1 & .019 & -1.0 & -2.4 & 0.3 & .130 & -1.2 & -3.1 & 0.6 & .200 & -1.1 & -2.6 & 0.5 & .180 \\
\hline
\end{tabular}

Model 1: unadjusted

Model 2: adjusted for age (squared)

Model 3: additionally adjusted for educational status, smoking, physical activity, alcohol consumption, comorbidities 
reduced the effect of lower physical HRQoL among all strata of metabolic health and obesity status compared to $\mathrm{MHNO}$, but remained statistically significant among both sexes. A difference of two to three points in the PCS is considered clinically relevant [20]. Thus, after adjusting for confounding variables lower values of physical HRQoL among MHO compared to MHNO remained clinically relevant only among women. Adjustment for confounding factors had the largest impact among MUNO as differences in comparison to MHNO do not remain clinically significant.

The strength of this study is its large sample of the general population which allows stratification for gender and adjustment for potential confounding variables. Furthermore, anthropometric parameters were measured by standard protocols and trained staff and valid data collection tools were used to assess physical HRQoL, diseases, and various health determinants, and biological parameters. A few limitations have to be acknowledged. Since this is a cross-sectional study the direction of the causal pathway between physical HRQoL and metabolic health according to obesity category cannot be identified. Poorer physical HRQoL, characterized by sedentary behaviour, might increase weight gain and vice versa. Due to the study design we used different cut-offs among fasting and non-fasting participants for serum glucose and triglycerides. Finally, bias due to misclassification cannot be ruled out completely.

\section{Conclusion}

Obesity was significantly related to lower physical HRQoL, independent of metabolic health status, which underlines previous findings that $\mathrm{MHO}$ status is not necessarily a healthy state. This inverse relationship can be partly explained by age, educational status, healthrelated behaviors, and comorbidities. The associations were found in both sexes and were consistently more pronounced among women compared to men.

\section{Additional files}

Additional file 1: Covariables (PDF $160 \mathrm{~kb}$ ).

Additional file 2: Sensitivity analyses with physical functioning as dependent variable (PDF $21 \mathrm{~kb}$ ).

\section{Abbreviations}

BMI: body mass index; HRQoL: health-related quality of life; MHNO: Metabolically healthy non-obese; MHO: Metabolically healthy obese; MUNO: Metabolically unhealthy non-obese; MUO: Metabolically unhealthy obese; PCS: physical component score; WC: waist circumference

\section{Acknowledgements}

Not applicable.

\section{Funding}

DEGS1 was primarily funded by the German Ministry of Health (BMG).

Supplementary funding was provided by the German Ministry of Food,
Agriculture, and Consumer Protection (BMELV) for monitoring of allergic sensitization, and by the German Association of Psychotherapy and Psychiatry (DGPPN) for implementing a module on mental health.

\section{Availability of data and materials}

The authors confirm that some access restrictions apply to the data underlying the findings. The data set cannot be made publicly available because informed consent from study participants did not cover public deposition of data. However, the minimal data set underlying the findings is archived in the 'Health Monitoring' Research Data Centre at the Robert Koch Institute (RKI) and can be accessed by all interested researchers. On-site access to the data set is possible at the Secure Data Center of the RKI's 'Health Monitoring' Research Data Centre. Requests should be submitted to the 'Health Monitoring' Research Data Centre, Robert Koch Institute, Berlin, Germany (e-mail: fdz@rki.de).

\section{Authors' contributions}

CSN, JT and AS designed the study. JT conducted the present analysis and AS, ABW, UH, GBMM, CSN contributed to the analysis plan and to the interpretation of the results. JT drafted the manuscript and AS, ABW, UH, GBMM, CSN critically revised it for important intellectual content. All authors read and approved the final manuscript. CSN, UH and GBMM were involved in the design and implementation of DEGS.

\section{Competing interests}

The authors declare that they have no competing interests.

Consent for publication

Not applicable.

\section{Ethics approval and consent to participate}

DEGS1 was approved by the Federal and State Commissioners for Data Protection and the Charité - Universitätsmedizin Berlin ethics committee (No. EA2/047/08). The implementation of the survey conforms to the principles of the Helsinki Declaration. All participants provided written informed consent prior to inclusion in the surveys.

\section{Publisher's Note}

Springer Nature remains neutral with regard to jurisdictional claims in published maps and institutional affiliations.

\section{Author details}

'Department of Epidemiology and Health Monitoring, Robert Koch Institute, Berlin, Germany. ${ }^{2}$ Institute of Nutritional Medicine, University of Hohenheim, Stuttgart, Germany.

Received: 24 November 2016 Accepted: 17 May 2017

Published online: 10 June 2017

\section{References}

1. Tepavcevic DK, Pekmezovic T, Stojsavljevic N, Kostic J, Basuroski ID, Mesaros $\mathrm{S}$, et al. Predictive value of health-related quality of life in progression of disability and depression in persons with multiple sclerosis: a 3-year study. Acta Neurol Belg. 2013;113:403-9.

2. UI-Haq Z, Mackay DF, Pell JP. Association between physical and mental health-related quality of life and adverse outcomes; a retrospective cohort study of 5,272 Scottish adults. BMC Public Health. 2014;14:1197.

3. Ul-Haq Z, Mackay DF, Fenwick E, Pell JP. Meta-analysis of the association between body mass index and health-related quality of life among adults, assessed by the SF-36. Obesity. 2013;21:E322-7.

4. Fontaine KR, Barofsky I. Obesity and health-related quality of life. Obes Rev. 2001;2:173-82.

5. Bentley TGK, Palta M, Paulsen AJ, Cherepanov D, Dunham NC, Feeny D, et al. Race and gender associations between obesity and nine health-related quality-of-life measures. Qual Life Res. 2011;20:665-74.

6. Audureau E, Pouchot J, Coste J. Gender-related differential effects of obesity on health-related quality of life via obesity-related Comorbidities: a mediation analysis of a French Nationwide survey. Circ Cardiovasc Qual Outcomes. 2016;9:246-56. 
7. Søltoft F, Hammer M, Kragh N. The association of body mass index and health-related quality of life in the general population: data from the 2003 health survey of England. Qual Life Res. 2009;18:1293-9.

8. Song HJ, Lee E-K, Kwon J-W. Gender differences in the impact of obesity on health-related quality of life. Asia Pac J Public Health. 2016;28:146-56.

9. Yang Y, Herting JR, Choi J. Obesity, metabolic abnormality, and healthrelated quality of life by gender: a cross-sectional study in Korean adults. Qual Life Res. 2016;25:1537-48.

10. Ul-Haq Z, Mackay DF, Fenwick E, Pell JP. Impact of metabolic comorbidity on the association between body mass index and health-related quality of life: a Scotland-wide cross-sectional study of 5,608 participants. BMC Public Health. 2012;12:143.

11. Lopez-Garcia E, Guallar-Castillon P, Garcia-Esquinas E, Rodriguez-Artalejo F. Metabolically healthy obesity and health-related quality of life: A prospective cohort study. Clin Nutr. 2016:36(3):853-860.

12. Wang J, Sereika SM, Styn MA, Burke LE. Factors associated with health related quality of life among overweight or obese adults. J Clin Nurs. 2013;22:2172-82.

13. Doll HA, Petersen SEK, Stewart-Brown SL. Obesity and physical and emotional well-being: associations between body mass index, chronic illness, and the physical and mental components of the SF-36 questionnaire. Obes Res. 2000:8:160-70.

14. Amiri P, Deihim T, Taherian R, Karimi M, Gharibzadeh S, Asghari-Jafarabadi $M$, et al. Factors affecting gender differences in the association between health-related quality of life and metabolic syndrome components: Tehran lipid and glucose study. PLoS One. 2015;10:e0143167.

15. Frisman GH, Kristenson M. Psychosocial status and health related quality of life in relation to the metabolic syndrome in a Swedish middle-aged population. Eur J Cardiovasc Nurs. 2009;8:207-15.

16. Scheidt-Nave C, Kamtsiuris P, Gößwald A, Hölling H, Lange M, Busch MA, et al. German health interview and examination survey for adults (DEGS) design, objectives and implementation of the first data collection wave. BMC Public Health. 2012;12:1-16.

17. Bullinger M. German translation and psychometric testing of the SF-36 health survey: preliminary results from the IQOLA project. International quality of life assessment. Soc Sci Med. 1995:41:1359-66.

18. Ware J. SF-36 health survey update. Spine (Phila Pa 1976). 2000;25:3130-9.

19. Ware J, Kosinski M, Bjorner J, Turner-Bowker D, Gandek B, Maruish M. User's manual for the SF-36v2 health survey. 2nd ed. Lincoln: Quality Metric Inc; 2007.

20. Ware J, Kosinski M, Keller S: SF-36 Physical and Mental Health Summary Scales: A User's Manual. Boston: Health Institute, New England Medical Center; 1994.

21. Ellert $U$, Kurth BM. Health related quality of life in adults in Germany: results of the German health interview and examination survey for adults (DEGS1). Bundesgesundheitsblatt Gesundheitsforschung Gesundheitsschutz. 2013;56:643-9.

22. Mensink GB, Schienkiewitz A, Haftenberger M, Lampert T, Ziese T, Scheidt-Nave C. Overweight and obesity in Germany: results of the German health interview and examination survey for adults (DEGS1). Bundesgesundheitsblatt Gesundheitsforschung Gesundheitsschutz. 2013;56:786-94.

23. World Health Organization. Obesity: preventing and managing the global epidemic. Geneva: WHO Technical Report Series 894; 2000.

24. Neuhauser H, Thamm M, Ellert U. Blood pressure in Germany 2008-2011: results of the German health interview and examination survey for adults (DEGS1). Bundesgesundheitsblatt Gesundheitsforschung Gesundheitsschutz. 2013;56:795-801

25. Knopf H, Grams D. Medication use of adults in Germany: results of the German health interview and examination survey for adults (DEGS1). Bundesgesundheitsblatt Gesundheitsforschung Gesundheitsschutz. 2013; 56:868-77.

26. van Vliet-Ostaptchouk J, Nuotio M-L, Slagter S, Doiron D, Fischer K, Foco L, et al. The prevalence of metabolic syndrome and metabolically healthy obesity in Europe: a collaborative analysis of ten large cohort studies. BMC Endocr Disord. 2014;14:9.

27. Truthmann J, Mensink GBM, Bosy-Westphal A, Scheidt-Nave C, Schienkiewitz A. Metabolic health in relation to body size: changes in prevalence over time between 1997-99 and 2008-11 in Germany. PLoS One. 2016;11:e0167159.

28. Expert Panel on Detection Evaluation and Treatment of High Blood Cholesterol in Adults. Executive summary of the third report of the national cholesterol education program (ncep) expert panel on detection, evaluation, and treatment of high blood cholesterol in adults (adult treatment panel iii). JAMA. 2001:285:2486-97.

29. Korhonen PE, Seppala T, Jarvenpaa S, Kautiainen H. Body mass index and health-related quality of life in apparently healthy individuals. Qual Life Res. 2014;23:67-74.

30. Eckel N, Meidtner K, Kalle-Uhlmann T, Stefan N, Schulze MB. Metabolically healthy obesity and cardiovascular events: a systematic review and meta-analysis. Eur J Prev Cardiol. 2016;23:956-66.

31. Soriguer F, Gutiérrez-Repiso C, Rubio-Martín E, García-Fuentes E, Almaraz MC, Colomo N. Antonio IEd, Adana MSRd, Chaves FJ, Morcillo S, et al. metabolically healthy but obese, a matter of time? Findings from the prospective Pizarra study. J Clin Endocrinol Metab. 2013;98:2318-25.

32. Hinnouho G-M, Czernichow S, Dugravot A, Batty GD, Kivimaki M, SinghManoux A. Metabolically healthy obesity and risk of mortality: does the definition of metabolic health matter? Diabetes Care. 2013:36:2294-300.

33. Roberson LL, Aneni EC, Maziak W, Agatston A, Feldman T, Rouseff M, et al. Beyond BMl: the "metabolically healthy obese" phenotype \& its association with clinical/subclinical cardiovascular disease and all-cause mortality - a systematic review. BMC Public Health. 2014;14:14.

34. Donini LM, Merola G, Poggiogalle E, Lubrano C, Gnessi L, Mariani S, Migliaccio S, Lenzi A. Disability, Physical Inactivity, and Impaired HealthRelated Quality of Life Are Not Different in Metabolically Healthy vs. Unhealthy Obese Subjects. Nutrients. 2016;8:E759.

35. Schienkiewitz A, Mensink GB, Scheidt-Nave C. Comorbidity of overweight and obesity in a nationally representative sample of German adults aged 18-79 years. BMC Public Health. 2012:12:658.

36. Krug S, Jordan S, Mensink G, Müters S, Finger J, Lampert T. Physical activity results of the German health interview and examination survey for adults (DEGS1). Epidemiologie Gesundheitsberichterstattung. 2013;56:765.

37. Cruz LN, Fleck MP, Oliveira MR, Camey SA, Hoffmann JF, Bagattini AM, et al. Health-related quality of life in Brazil: normative data for the SF-36 in a general population sample in the south of the country. Cien Saude Colet. 2013:18:1911-21.

38. Sullivan M, Karlsson J. The Swedish SF-36 health survey III. Evaluation of criterion-based validity: results from normative population. J Clin Epidemiol. 1998;51:1105-13.

\section{Submit your next manuscript to BioMed Central and we will help you at every step:}

- We accept pre-submission inquiries

- Our selector tool helps you to find the most relevant journal

- We provide round the clock customer support

- Convenient online submission

- Thorough peer review

- Inclusion in PubMed and all major indexing services

- Maximum visibility for your research

Submit your manuscript at www.biomedcentral.com/submit 